\title{
SHELTERED MODULES AND RINGS ${ }^{1}$
}

\author{
SETH WARNER
}

ABstract. The problem of determining when a module (ring) admits an indiscrete, Hausdorff linear (ideal) topology is discussed in terms of sheltered modules (rings).

Our purpose here is simply to point out the relevance of the notion of sheltered submodule (ideal), introduced by Leptin [4, p. 245], to the problem of determining when a module (ring) admits a proper linear (ideal) topology, recently discussed by Hochster [3]. The topology of a topological module is linear if the open submodules form a fundamental system of neighborhoods of zero; a linear topology is proper if it is Hausdorff but not discrete. Similarly, the topology of a ring is an ideal topology if the open ideals form a fundamental system of neighborhoods of zero; an ideal topology is proper if it is Hausdorff but not discrete.

A proper submodule $U$ of a (not necessarily unitary) module $E$ is sheltered [4, p. 245], [2, Exercise 18, p. 110] if the set of submodules of $E$ strictly containing $U$ has a smallest member $S$, called the shelter of $U$. A module $E$ is a sheltered module if $(0)$ is a sheltered submodule of $E$. If $U$ is a proper submodule of $E$, then $E / U$ is a sheltered module if and only if $U$ is a sheltered submodule of $E$. A sheltered module clearly admits no proper linear topology; one of our purposes is to determine the relationship between modules admitting no proper linear topology and sheltered modules.

The definition suggests that a sheltered submodule is in some sense large. Indeed, a maximal submodule is clearly sheltered; in a vector space the sheltered subspaces are precisely those of codimension 1 ; more generally, the sheltered submodules of a semisimple module are precisely the maximal ones by [1, Theorem 1, p. 32]. In general, if $U$ is a submodule and if $x \notin U$, then clearly $U$ is a sheltered submodule and $x$ belongs to its shelter if and only if $U$ is maximal in the set of all submodules not containing $x$, in which case its shelter is the submodule generated by $U$ and $x$. Consequently by Zorn's lemma, every

Received by the editors November 10, 1970 .

AMS 1970 subject classifications. Primary 16A64, 16A80.

Key words and phrases. Sheltered module, sheltered submodule, sheltered ring, sheltered ideal, topological module, topological ring.

${ }^{1}$ Written while the author was in residence at Reed College. 
submodule is the intersection of sheltered submodules [2, Exercise 18(a), p. 110]. Therefore, the filter base generated by all sheltered submodules is a fundamental system of neighborhoods for a Hausdorff linear topology; consequently, if $E$ admits no proper linear topology, $(0)$ is the intersection of finitely many sheltered submodules.

TheOREM 1. Let $E$ be a nonzero module. The following statements are equivalent:

$1^{\circ}$. E admits no proper linear topology.

$2^{\circ}$. There exist sheltered submodules $U_{1}, \cdots, U_{n}$ of $E$ such that $U_{1} \cap \cdots \cap U_{n}=(0)$.

$3^{\circ}$. E is isomorphic to a submodule of the cartesian product of finitely many sheltered modules.

Proof. We have just seen that $1^{\circ}$ implies $2^{\circ}$. If $2^{\circ}$ holds, $E$ is isomorphic to a submodule of $\prod_{i=1}^{n}\left(E / U_{i}\right)$, so $3^{\circ}$ holds. If a module admits no proper linear topology, neither does any submodule (for otherwise the filter of neighborhoods of zero of the submodule would be a fundamental system of neighborhoods of zero for a proper linear topology on the module). As a sheltered module admits no proper linear topology, to show that $3^{\circ}$ implies $1^{\circ}$ it suffices to show that the cartesian product of modules admitting no proper linear topology also admits no proper linear topology; a proof of this fact is a simplification of the proof of the following theorem, which in turn is essentially the proof of $[3$, Theorem 2].

THEOREM 2. A subdirect sum of finitely many rings admitting no proper ideal topology also admits no proper ideal topology.

Proof. If $A$ is a subdirect sum of $A_{1}, \cdots, A_{n}$ and if $\pi$ is the canonical projection from $\prod_{i=1}^{n} A_{i}$ on to $\prod_{i=1}^{n-1} A_{i}$, then $A$ is a subdirect sum of $\pi(A)$ and $A_{n}$. Hence by induction it suffices to consider the case $n=2$. If $V$ is an ideal of $A$, then $V_{1}=\left\{x \in A_{1}:(x, 0) \in V\right\}$ is an ideal of $A_{1}$, for if $a \in A_{1}$ and $x \in V_{1}$, then there exists $y \in A_{2}$ such that $(a, y) \in A$, whence $(a x, 0)=(a, y)(x, 0) \in V$ and similarly $(x a, 0) \in V$, and thus $a x, x a \in V_{1}$. The proof now proceeds like the first half of the proof of [3, Theorem 2].

An artinian module $E$ admits no proper linear topology, since for any linear topology on $E$ there is a minimal open submodule. The prominence of sheltered submodules in an artinian module is indicated in the following theorem.

THEOREM 3. A unitary module $E$ is artinian if and only if each proper submodule of $E$ is the intersection of finitely many sheltered submodules. 
Proof. If $M, U$, and $S$ are submodules such that $M \subseteq U \subseteq S$, then clearly $U$ is a sheltered submodule with shelter $S$ if and only if $U / M$ is a sheltered submodule of $E / M$ with shelter $S / M$. The condition is therefore necessary by Theorem 1 , since a quotient module of an artinian module is artinian. Conversely, if $M$ is the intersection of a decreasing sequence of submodules, the condition implies that $E / M$ admits no proper linear topology and consequently that the sequence is stationary.

THEOREM 4. If $E$ is a module, every proper submodule of $E$ is sheltered if and only if the set of all submodules of $E$ is well ordered by inclusion.

Proof. Necessity. Let $\mathfrak{T}$ be a nonempty set of submodules, and let $N=\cap\{M: M \in \mathfrak{T}\}$. If $N \notin \mathfrak{T}$, then every member of $\mathfrak{T}$ properly contains $N$, so $N$ is proper and every member of $\mathscr{T}$ contains the shelter $S$ of $N$, whence $N \supseteq S \supset N$, a contradiction. Thus every nonempty set of submodules contains a smallest member.

Sufficiency. If $M$ is a proper submodule, the smallest of the submodules strictly containing $N$ is clearly the shelter of $M$.

If $A$ is a ring, then the ideals of $A$ are precisely the submodules of $A$, regarded as a module over the subring of endomorphisms of the additive group of $A$ generated by right and left multiplications. Applying the general definition to this situation, we call an ideal $\mathfrak{u}$ of $A$ sheltered if the set of all ideals strictly containing $\mathfrak{u}$ has a smallest member $\mathfrak{z}$, called the shelter of $\mathfrak{u} ; A$ is a sheltered ring if (0) is a sheltered ideal of $A$. Clearly a sheltered ring admits no proper ideal topology.

TheOREM 5. Let $A$ be a nonzero ring. The following statements are equivalent:

$1^{\circ}$. A admits no proper ideal topology.

$2^{\circ}$. There exist sheltered ideals $\mathfrak{u}_{1}, \cdots, \mathfrak{u}_{n}$ of $A$ such that $\mathfrak{u}_{1} \cap \cdots \cap$ $\mathfrak{u}_{n}=(0)$.

$3^{\circ}$. $A$ is isomorphic to a subdirect sum of finitely many sheltered rings.

Proof. By Theorem $1,1^{\circ}$ implies $2^{\circ}$ and $2^{\circ}$ implies $3^{\circ}$. By Theorem $2,3^{\circ}$ implies $1^{\circ}$.

In [3, Theorem 1] Hochster characterized those commutative rings with identity that admit no proper ideal topology in terms of the annihilators of their maximal ideals. We shall determine those maximal ideals having nonzero annihilators and their annihilators in terms of a minimal family of sheltered ideals whose intersection is (0) and their shelters, first for sheltered rings and then in general; this will make evident once again the necessity of the conditions given in [3, Theorem 1]. 
THEOREM 6. Let $A$ be a sheltered commutative ring with identity, let 8 be the shelter of (0), and let $\mathrm{m}=\mathrm{Ann} 8$. Then $\mathrm{m}$ is a maximal ideal and consists of all zero-divisors of $A$, and $=$ Ann $m$; in particular, Ann $m$ is a principal ideal. If $A$ is noetherian, then $A$ is artinian and $\mathfrak{m}$ is its only maximal ideal.

Proof. Clearly $\mathfrak{m}$ is a proper ideal. To show that $\mathfrak{m}$ is maximal, let $a \notin m$. Then there exists $s \in \mathbb{z}$ such that $a s \neq 0$, so $A a s \cap \mathbb{z} \neq(0)$, whence $A a s=8$. In particular, there exists $b \in A$ such that $b a s=s$, so $(b a-1) s=0$; the annihilator of $b a-1$ is, therefore, a nonzero ideal, so it contains $\mathfrak{B}$, and consequently $b a-1 \in A n n \mathfrak{z}=\mathfrak{m}$. Thus $A / \mathfrak{m}$ is a field, so $\mathfrak{m}$ is maximal. By definition, every element of $\mathfrak{m}$ is a zerodivisor. Conversely, if $c$ is a zero-divisor, then its annihilator is a nonzero ideal and hence contains $\mathfrak{z}$, so $c \in A$ Ann $z=\mathfrak{m}$.

We may regard Ann $\mathfrak{m}$ as a vector space over $A / \mathfrak{m}$; the subspaces of the $(A / \mathfrak{m})$-vector space Ann $\mathfrak{m}$ are precisely the ideals of $A$ contained in Ann $\mathfrak{m}$; therefore Ann $\mathfrak{m}$ has a smallest nonzero subspace $\mathbb{B}$, so Ann $m=8$. The final assertion follows from the proof of $[3$, Theorem 4]. Alternatively, we may observe that $\bigcap_{n=1}^{\infty} \mathrm{m}^{n}=(0)$ by Krull's theorem [5, Theorem 12 , p. 216], whence $\mathrm{m}^{n}=(0)$ for some $n \geqq 1$ as $A$ admits no proper ideal topology; therefore as $A / \mathrm{m}$ is a field, $A$ is artinian by [1, Proposition 12, p. 71]; as $\mathfrak{m}$ is nilpotent, $\mathfrak{m}$ is contained in the radical of $A$, so $\mathrm{m}$ is the only maximal ideal of $A$.

THEOREM 7. Let $A$ be a commutative ring with identity that admits no proper ideal topology. Let $\mathfrak{u}_{1}, \cdots, \mathfrak{u}_{n}$ be sheltered ideals of $A$ such that $\bigcap_{i=1}^{n} \mathfrak{u}_{i}=(0)$ but for each $i \in[1, n], \mathfrak{u}_{i} \Phi \bigcap_{j \neq i} \mathfrak{u}_{j}$. For each $i \in[1, n]$ let $\mathfrak{B}_{i}$ be the shelter of $\mathfrak{u}_{i}$, let $\mathfrak{m}_{i}=\left(\mathfrak{u}_{i}: \mathfrak{B}_{i}\right)$, let $K(i)=\left\{j \in[1, n]: \mathfrak{m}_{j}=\mathfrak{m}_{i}\right\}$, and let $k_{i}$ be the number of integers in $K(i)$.

$1^{\circ}$. The maximal ideals of $A$ having nonzero annihilators are precisely the ideals $\mathfrak{m}_{1}, \cdots, \mathfrak{m}_{n}$, and $\bigcup_{k=1}^{n} \mathfrak{m}_{k}$ is the set of all zero-divisors of $A$.

$2^{\circ}$. Ann $\mathfrak{m}_{i}=\left(\bigcap_{j \in K(i)} g_{j}\right) \cap\left(\bigcap_{j \notin K(i)} \mathfrak{u}_{j}\right)$, a nonzero ideal generated by not more than $k_{i}$ elements.

$3^{\circ}$. For each nonzero $a \in A$ there exists $i \in[1, n]$ such that $A a \cap$ Ann $\mathfrak{m}_{i} \neq(0)$.

$4^{\circ}$. If $A$ is noetherian, then $A$ is artinian and $\mathfrak{m}_{1}, \cdots, \mathfrak{m}_{n}$ are its only maximal ideals.

Proof. Let $\phi_{i}$ be the canonical epimorphism from $A$ onto the sheltered ring $A / \mathfrak{u}_{i}$. Clearly $\phi_{i}\left(\mathfrak{m}_{i}\right)=$ Ann $\phi_{i}\left(\mathbb{g}_{i}\right)$, so, by Theorem 6 , $\phi_{i}\left(\mathfrak{m}_{i}\right)$ is a maximal ideal of $A / \mathfrak{u}_{i}$ and its annihilator is $\phi_{j}\left(\mathfrak{g}_{j}\right)$. As $\mathfrak{m}_{i} \supseteq \mathfrak{u}_{i}$, therefore, $\mathfrak{m}_{i}$ is a maximal ideal of $A$. Let $x \in A n n \mathfrak{m}_{i}$. Then $\phi_{j}(x) \in$ Ann $\phi_{j}\left(\mathfrak{m}_{i}\right)$ for all $j \in[1, n]$; if $j \in K(i)$, then $\phi_{j}(x) \in \phi_{j}\left(\mathfrak{z}_{j}\right)$ by 
Theorem 6, whence $x \in \mathfrak{g}_{j}$; if $j \notin K(i)$, then $\mathfrak{m}_{i}+\mathfrak{m}_{j}=A$, so $\phi_{j}\left(\mathfrak{m}_{i}\right)$ $=A / \mathfrak{u}_{j}$ and hence has a zero annihilator, whence $x \in \mathfrak{u}_{j}$. Thus Annm $\mathfrak{m}_{i}$ $\subseteq\left(\bigcap_{j \in K(i)} \mathbb{B}_{j}\right) \cap\left(\bigcap_{j \notin K(i)} \mathfrak{u}_{j}\right)$. Conversely, if $x \in \mathbb{z}_{j}$ where $j \in K(i)$, then $\mathfrak{m}_{i} x=\mathfrak{m}_{j} x \subseteq \mathfrak{u}_{j}$; hence if $x \in\left(\bigcap_{j \in K(i)} \mathfrak{B}_{j}\right) \cap\left(\bigcap_{j \notin K(i)} \mathfrak{u}_{j}\right)$, then $\mathfrak{m}_{i} x$ $\subseteq \bigcap_{k=1}^{n} \mathfrak{u}_{k}=(0)$, so $x \in$ Annm $_{i}$. Therefore Ann $\mathfrak{m}_{i}=\left(\bigcap_{j \in K(i)} \vec{z}_{j}\right)$ $\bigcap\left(\bigcap_{j \notin K(i)} \mathfrak{u}_{j}\right)$. This ideal is nonzero, since $\mathfrak{B}_{i} \cap\left(\bigcap_{j \neq i} \mathfrak{u}_{j}\right) \neq(0)$; indeed, as $\mathfrak{u}_{i} \Phi \bigcap_{j \neq i} \mathfrak{u}_{j}, \mathfrak{u}_{i}+\bigcap_{j \neq i} \mathfrak{u}_{j} \supseteq \mathfrak{Z}_{i}$; let $s \in \mathfrak{B}_{i}, s \notin \mathfrak{u}_{i}$; then $s=u+v$ where $u \in \mathfrak{u}_{i}$ and $v \in \bigcap_{j \neq i} \mathfrak{u}_{j}$, so $v=s-u \in \mathfrak{B}_{i} \cap\left(\bigcap_{j \neq i} \mathfrak{u}_{j}\right)$ and $v \neq 0$. By [5, Remark, p. 215], to complete the proof of $1^{\circ}$ it suffices to show that every zero-divisor $a$ of $A$ belongs to some $\mathfrak{m}_{i}$. Let $a x=0$ where $x \neq 0$; then $x \notin \mathfrak{u}_{i}$ for some $i$, so $\phi_{i}(a)$ is a zero-divisor of $A / \mathfrak{u}_{i}$, whence $\phi_{i}(a)$ $\in \phi_{i}\left(\mathfrak{m}_{i}\right)$ by Theorem 6 and thus $a \in \mathfrak{m}_{i}$.

To complete the proof of $2^{\circ}$, we note that the canonical mappings of the $\left(A / \mathrm{m}_{i}\right)$-vector spaces

$$
\begin{aligned}
\operatorname{Ann} \mathfrak{m}_{i}=\left(\bigcap_{j \in K(i)} \mathfrak{B}_{j}\right) \cap\left(\bigcap_{j \notin K(i)} \mathfrak{u}_{j}\right) \\
\rightarrow\left(\bigcap_{j \in K(i)} \mathfrak{B}_{j}\right) /\left(\bigcap_{j \in K(i)} \mathfrak{u}_{j}\right) \rightarrow \prod_{j \in K(i)}\left(\mathbb{g}_{j} / \mathfrak{u}_{j}\right)
\end{aligned}
$$

are monomorphisms; by Theorem $6, \mathfrak{B}_{j} / \mathfrak{u}_{j}$ has dimension 1 over $A / \mathfrak{m}_{i}$ for each $j \in K(i)$, so Ann $\mathfrak{m}_{i}$ has dimension $\leqq k_{i}$; thus $2^{\circ}$ holds.

Statement $3^{\circ}$ follows from $1^{\circ}$ and [3, Theorem 1]; we give a proof not involving Zorn's lemma. First, if $b \neq 0$, then $A b \cap z_{i} \neq(0)$; indeed, the assertion is clear if $b \in \mathfrak{l}_{i}$; otherwise $A b+\mathfrak{u}_{i} \supseteq \mathfrak{z}_{i}$, so if $s \in \mathfrak{B}_{i}$ but $s \notin \mathfrak{u}_{i}$, then $x b+u=s$ for some $u \in \mathfrak{u}_{i}$, whence $x b=s-u$, a nonzero element of $\xi_{i}$. Therefore if $a \neq 0$, there exists $x_{1} \in A$ such that $x_{1} a \in g_{1}$ and $x_{1} a \neq 0$; hence there exists $x_{2} \in A$ such that $x_{2}\left(x_{1} a\right) \in \mathbb{B}_{1} \cap \varepsilon_{2}$ and $x_{2}\left(x_{1} a\right)$ $\neq 0$, etc.; in sum, $A a \cap\left(\bigcap_{i=1}^{n} B_{i}\right) \neq(0)$. To prove $3^{\circ}$, therefore, we may assume that $a \in \bigcap_{i=1}^{n} B_{i}$. If $a \in A n n m_{1}$, then there exists $y_{1} \in \mathfrak{m}_{1}$ such that $y_{1} a \neq 0$ and $y_{1} a \in \mathfrak{u}_{1}$ as $a \in \mathbb{z}_{1}$; if $y_{1} a \notin A n n m_{2}$, there exists $y_{2} \in \mathfrak{m}_{2}$ such that $y_{2}\left(y_{1} a\right) \neq 0$ and $y_{2}\left(y_{1} a\right) \in \mathfrak{u}_{2} \cap \mathfrak{u}_{1}$ as $y_{1} a \in \mathcal{B}_{2}$; continuing in this way, we arrive at $r<n$ such that $y_{r} \cdots y_{1} a \neq 0$ and $y_{r} \cdots y_{1} a \in$ Annm $\mathfrak{m}_{r+1}$, for otherwise $y_{n} y_{n-1} \cdots y_{1} a \neq 0$ but $y_{n} \cdots y_{1} a \in \mathfrak{u}_{n} \cap \cdots$ $\cap \mathfrak{u}_{1}=(0)$, a contradiction.

Again, $4^{\circ}$ follows from $1^{\circ}$ and the proof of [3, Theorem 4]; it also follows from Theorem 6 since every maximal ideal $m$ must contain some $\mathfrak{u}_{i}$ (otherwise $\mathfrak{m}+\mathfrak{u}_{i}=A$ for all $i$, so $\mathfrak{m}=\mathfrak{m}+\bigcap_{i=1}^{n} \mathfrak{u}_{i}=A$, a contradiction), so if $A$ is noetherian, then $\mathfrak{m}_{1}, \cdots, \mathfrak{m}_{n}$ are the only maximal ideals by Theorem 6 . Also by Theorem 6 , each $\phi_{i}\left(\mathfrak{m}_{i}\right)$ is nilpotent, so the radical of $A$ is also as $\bigcap_{i=1}^{n} \mathfrak{u}_{i}=(0)$, and therefore $A$ is artinian. 
In [2, Exercise 17(b), p. 11] it is incorrectly asserted that a module admitting no proper linear topology is artinian. Leptin $[4, \mathrm{p}$. 248] gave an example of a nonartinian, sheltered module. Here is an example of a commutative, nonnoetherian, sheltered ring. Let $B$ be a basis of an infinite-dimensional vector space $A$ over a field $K$, let $e, z$ be two elements of $B$, and let $C=B-\{e, z\}$. To define a multiplication on $A$ we indicate the product of elements of $B$ as follows: $e b=b e$ $=b$ for all $b \in B$; for all $c, d \in C, c d=d c=z$ if $c \neq d$ and $c^{2}=0 ; c z=z c$ $=z^{2}=0$ for all $c \in C$. Clearly $A$ is a commutative ring with identity $e$, and the subspace $\mathfrak{m}$ of codimension 1 generated by $C \cup\{z\}$ is a maximal ideal satisfying $\mathfrak{m}^{3}=(0)$. Consequently, $\mathfrak{m}$ is contained in the radical of $A$, so $\mathrm{m}$ is the only maximal ideal of $A$, i.e., $A$ is a local ring. To show that $z \in A a$ if $a \neq 0$, let $a=\sum_{b \in B} \lambda_{b} b$ and let $\mu=\sum_{c \in C} \lambda_{c}$. If $\lambda_{e} \neq 0$, then $\lambda_{e}^{-1} z a=z$; if $\lambda_{e}=0$ and $\mu \neq 0$, then $\mu^{-1} d a=z$ where $d \in C$ is such that $\lambda_{d}=0$; if $\lambda_{e}=\mu=0$, then either $\lambda_{c} \neq 0$ for some $c \in C$, in which case $\left(-\lambda_{c}\right)^{-1} c a=z$, or else $\lambda_{c}=0$ for all $c \in C$, in which case $a=\lambda_{z} z$ and thus $\lambda_{z}^{-1} a=z$. From this it follows that $K z$ is the shelter of (0) and that $A a=K a+K z$ for all nonzero $a \in \mathfrak{m}$; thus $\mathfrak{m}$ is not a finitely generated ideal.

We may characterize those sheltered commutative rings with identity in which the annihilator of the shelter of $(0)$ is nilpotent (such as the ring of the preceding example) as follows:

TheORem 8. Let $A$ be a commutative ring with identity. Then $A$ has a nilpotent maximal ideal $\mathrm{m}$ whose annihilator $\mathrm{z}$ is a principal ideal if and only if $A$ is a sheltered ring and there exists an integer $n$ such that the product of any sequence of $n$ zero-divisors is zero.

Proof. The condition is sufficient by Theorem 6; we need only let $\mathfrak{m}$ be the annihilator of the shelter of $(0)$.

Necessity. Let $\mathfrak{m}^{n}=(0)$. Clearly $\mathbb{z} \neq(0)$. We may regard $\mathfrak{z}$ as a onedimensional vector space over $A / \mathrm{m}$ since $B$ is principal. Since any ideal of $A$ contained in $\mathbb{B}$ is a subspace of the $(A / \mathrm{m})$-vector space $\mathbb{B}$, we conclude that is a minimal ideal. Let $a$ be a nonzero element of $A$, and let $r \in[0, n-1]$ be such that $a \mathfrak{m}^{r} \neq(0)$ but $a \mathfrak{m}^{r+1}=(0)$; let $x \in \mathfrak{m}^{r}$ be such that $a x \neq 0$; then $a x \mathfrak{m}=(0)$, so $a x \in \mathbb{z}$. Thus $A a \cap z \neq(0)$, so as $\mathfrak{B}$ is minimal, $A a \supseteq \mathfrak{z}$. Thus $\mathbb{z}$ is the shelter of $(0)$. Consequently by Theorem $6, \mathrm{~m}$ is the set of all zero-divisors, so the product of any $n$ zero-divisors is zero.

A question suggested by $3^{\circ}$ of Theorem 5 is: When is a commutative ring $A$ with identity that admits no proper ideal topology isomorphic to the cartesian product of finitely many sheltered rings? It follows from Theorem 6 that a necessary condition for $A$ to be iso- 
morphic to the cartesian product of finitely many sheltered rings is that the annihilator of every maximal ideal of $A$ be a principal ideal. This condition is also sufficient if $A$ is noetherian (Theorem 10).

THEOREM 9. Let $A$ be a commutative ring with identity whose radical is nilpotent. Then $A$ is isomorphic to the cartesian product of finitely many local sheltered rings if and only if $A$ has only finitely many maximal ideals, and the annihilator of each maximal ideal is principal.

Proof. Sufficiency. Let $\mathfrak{m}_{1}, \cdots, \mathfrak{m}_{n}$ be the maximal ideals of $A$. The radical $\mathfrak{r}$ of $A$ is $\mathfrak{m}_{1} \cdots \mathfrak{m}_{n}$, so $(0)=\mathfrak{r}^{k}=\mathfrak{m}_{1}^{k} \cdots \mathfrak{m}_{n}^{k}=\mathfrak{m}_{1}^{k} \cap \cdots$ $\cap \mathrm{m}_{n}^{k}$ for some $k$. Let $A_{i}=A / \mathrm{m}_{i}^{k}, 1 \leqq i \leqq n$, and let $\phi_{i}$ be the canonical epimorphism from $A$ on to $A_{i}$. Clearly $A_{i}$ is a local ring whose maximal ideal is nilpotent, and $A$ is canonically isomorphic to $\prod_{i=1}^{n} A_{i}[\mathbf{5}$, Theorem 32, p. 178]. By Theorem 8, it therefore suffices to prove that Ann $\phi_{i}\left(m_{i}\right)$ is a principal ideal of $A_{i}$. For this, we need only show that $\phi_{i}\left(\right.$ Ann $\left.\mathfrak{m}_{i}\right)=$ Ann $\phi_{i}\left(\mathfrak{m}_{i}\right)$, since Ann $\mathfrak{m}_{i}$ is principal by hypothesis. Clearly $\phi_{i}\left(\right.$ Ann $\left.\mathfrak{m}_{i}\right) \subseteq$ Ann $\phi_{i}\left(\mathfrak{m}_{i}\right)$. Conversely, let $\phi_{i}(x) \in \operatorname{Ann} \phi_{i}\left(\mathfrak{m}_{i}\right)$. Then $x=y+z$ where $y \in \mathrm{m}_{i}^{k}$ and $z \in \prod_{j \neq i} \mathrm{~m}_{j}^{k}$ [5, Theorem 31, p. 177], whence $\phi_{i}(x)=\phi_{i}(z)$; as $x \mathfrak{m}_{i} \subseteq \mathfrak{m}_{i}^{k}, z \mathfrak{m}_{i} \subseteq x \mathfrak{m}_{i}+y \mathfrak{m}_{i} \subseteq \mathfrak{m}_{i}^{k}$, so $z \mathfrak{m}_{i} \subseteq \mathfrak{m}_{i}^{k}$ $\cap \prod_{j \neq i} \mathrm{~m}_{j}^{k}=\prod_{j=1}^{n} \mathrm{~m}_{j}^{k}=(0)$; thus $\phi_{i}(x)=\phi_{i}(z) \in \phi_{i}\left(\right.$ Ann $\left.\mathfrak{m}_{i}\right)$.

THEOREM 10. Let $A$ be a commutative noetherian ring that admits no proper ideal topology. Then $A$ is isomorphic to the cartesian product of finitely many sheltered rings if and only if the annihilator of each maximal ideal of $A$ is principal.

Proof. By $4^{\circ}$ of Theorem $7, A$ has only finitely many maximal ideals and its radical is nilpotent. The condition is therefore sufficient by Theorem 9 .

\section{REFERENCES}

1. N. Bourbaki, Algèbre. Chap. 8, Actualités Sci. Indust., no. 1261, Hermann, Paris, 1958. MR $20 \# 4576$.

2. - Algèbre commutative. Chaps. 3, 4, Actualités Sci. Indust., no. 1293, Hermann, Paris, 1961. MR 30 \#2027.

3. M. Hochster, Rings with nondiscrete ideal topologies, Proc. Amer. Math. Soc. 21 (1969), 357-362. MR 39 \#195.

4. Horst Leptin, Linear kompakte Moduln und Ringe, Math. Z. 62 (1955), 241267. MR 16, 1085.

5. Oscar Zariski and Pierre Samuel, Commutative algebra. Vol. I, Van Nostrand, Princeton, N. J., 1958. MR 19, 833.

Duke University, Durham, North Carolina 27706 